\title{
On the Rank Minimization Problem
}

\author{
Yoonsoo Kim and Mehran Mesbahi
}

\begin{abstract}
After a brief overview of the problem of finding the extremal (minimum or maximum) rank positive semidefinite matrix subject to matrix inequalities, we identify a few new classes of such problems that can be efficiently solved. We then proceed to present an algorithm for solving the general class of rank minimization problems.

Index Terms - Rank minimization under LMI constraints, semidefinite programming, nonconvex quadratically constrained quadratic programs
\end{abstract}

\section{INTRODUCTION}

We consider the problem of minimizing or maximizing the rank of a positive semidefinite matrix over a set defined by linear matrix inequalities; we will refer to this class of problems as the rank minimization (or maximization) problem and denote them collectively by RMP. As a satisfactory characterization of the solution set of the general RMP is currently not available, and more to the point, that the RMP is NP-hard, we first restrict our attention to particular instances of the RMP in this paper. Specifically, our attempt will be on deriving sufficient conditions that guarantee the rank feasibility of a system of matrix inequalitiesfollowed by the reduction of a class of RMPs to semidefinite programs (SDP). We then proceed to propose an algorithm to solve general RMPs with a guaranteed convergence behavior- albeit possibly a running time that is exponential in the dimension of the problem.

\section{A. Motivation}

It has now been recognized that in spite of the many successes of linear matrix inequalities and semidefinite programming in resolving system and control problems, that there an many instances where the approach has sever limitations. Some important examples of such LMIimpaired instances include control system design in face of structural constraints, such as a fixed order or a block diagonal structure (e.g., decentralized control) [9]. In a different context, although the linear algebraic approach in combinatorics has led to many elegant solution strategies for graph theoretic and combinatorial problems, there are many instances where the corresponding matrix equalities and inequalities have to be accompanied by a rank qualification on the solution set (see $\S \mathrm{IV}$ ). The purpose of the present paper is three-fold: providing a brief overview of the RMP related research, identifying few new classes of RMPs that can be efficiently solved or characterized, and then

\footnotetext{
March 2004. The research of the authors was supported by the National Science Foundation NSF/CMS-0093456.

The authors are with the Department of Aeronautics and Astronautics, University of Washington, Seattle, WA. Emails: yoon-
} soo@aa.washington.edu and mesbahi@aa.washington.edu proceeding to propose an algorithm for general RMPs. A numerical example on RMPs related to the graph realization problem concludes the paper.

First, a few words on notation and background. We denote the space of $n \times n$ real symmetric matrices by $\mathcal{S}^{n}$ and its positive semidefinite subset by $\mathcal{S}_{+}^{n} ; I_{n}$ will denote the $n \times n$ identity matrix. For a vector $x$, Diag $(x)$ denotes the diagonal matrix of appropriate dimensions with $x$ on its diagonal. We note that $\mathcal{S}^{n}$ when equipped with the trace inner product $\langle.,\rangle:. \mathcal{S}^{n} \times \mathcal{S}^{n} \rightarrow \mathbf{R}$ is a finite dimensional inner product space. Moreover, the pointed closed convex cone $\mathcal{S}_{+}^{n}$ can be employed to define an order on $\mathcal{S}^{n}$, referred to as the positive-semidefinite ordering. In this venue, for two matrices $A, B \in \mathcal{S}^{n}$, we write $A \geq B$ if and only if the matrix difference $A-B$ is positive semidefinite. Two operations on the cone $\mathcal{S}_{+}^{n}$ are of our particular interest in this paper, namely, the integer-valued rank function rank $\mathcal{S}_{+}^{n} \rightarrow\{0,1, \ldots, n\}$ and the trace functional trace $\mathcal{S}_{+}^{n} \rightarrow \Re_{+}$; the latter is a convex function on $\mathcal{S}_{+}^{n}$ while the former is clearly not convex. When a linear matrix inequality is augmented with a rank constraint, the resulting feasibility or optimization problem often becomes much more difficult to solve. For example, rank constraints can be used to express integrality of the sought solutionthis observation provides a direct link between RMPs and integer programming. Minimizing or maximizing the trace of a positive semidefinite matrix subject to linear matrix inequalities, on the other hand, can be solved efficiently by the recently proposed interior point methods. Although minimizing the trace and the rank subject to LMI constraints are two different classes of problems, we note some obvious relationships between the two. For example, when the feasible matrices are known to have zero-one eigenvalues, the two optimization problems are clearly equivalent.

As it was pointed above, the RMP provides an interesting framework to resolve some of the outstanding problems in system and control synthesis. The RMPs are also of central importance in other areas of system theory, to name a few, in estimation theory (minimal order observers) and system identification (model reduction). However, the applications of RMPs go surprisingly further than system theory and control engineering to diverse areas of applied and pure mathematics such as differential geometry, statistics, cryptosystems, and combinatorial optimization.

\section{B. Related Works}

The earliest result on the RMPs is possibly due to Ledermann who encountered them in factorial analysis in statistics [25]. In his work, Ledermann is concerned with 
finding conditions under which the problem of minimizing the rank and minimizing the trace functional, over a very special class of linear matrix inequalities, are equivalent. In the same spirit, motivated by problems in reduced order synthesis, a restricted class of RMPs was considered in [26], and subsequently generalized in [27]. In both of these references, it was observed that in solving certain classes of the RMPs, the objective of minimizing the rank can be substituted by minimization of the trace. Consequently, these class of problems can be solved via for example, the interior point methods for semidefinite programming (SDP) [28]. Moreover, in [17], [31], it was further noted that some classes of the RMPs are in fact reducible to a system of matrix equations and thus, can be approached by a simple, non-interior point based, polynomial-time algorithms.

For general RMPs the solution strategies and theoretical analysis are rather limited. This is mainly due to the nonconvex nature of general RMPs and their NP-hardness [5]. It thus seems natural that one pursues the following directions in RMP research: (1) identify a wider class of RMPs that can be solved efficiently, either reducible to SDPs, or matrix equations, etc., (2) consider globally convergent RMP algorithms- with possibly exponential running times, (3) propose heuristic, approximate, or randomized algorithms, for general or special classes of RMPs. A recent example of the second approach via algebraic geometry can be found in [7]. Specifically, in [7] the RMP is reformulated as a finite (possibly exponentially large) sequence of semidefinite relaxations using the technique developed in [29]. In the same spirit but along a different venue, we will propose an algorithm for general RMPs in $\S$ III.

We conclude this section by briefly mentioning few RMP related research works that aim to find RMP algorithms with satisfactory practical performance. These methods include those based on minimizing a generalized trace of a matrix [10], [11], alternating projections methods [3], inversion of analytic centering [6], potential reduction methods [6], [20], coordinate descent methods [22], [33], linearization [14], [18], [21], and those based on the cutting plane algorithms [32].

\section{SOME SPECIAL CLASSES OF RMPS}

Closely related to the RMP is investigating the rank of feasible matrices satisfying a system of matrix inequalities. In this venue, let us consider the set of matrices $A_{i} \in \mathcal{S}_{+}^{d}$ and $b_{i} \in \mathbf{R}(i=1, \ldots, m)$, and let

$$
\begin{aligned}
\mathcal{F}\left(A_{1}, \ldots, A_{m}, b\right):= & \left\{X \in \mathcal{S}_{+}^{d} \mid\left\langle A_{i}, X\right\rangle \geq b_{i},\right. \\
& i=1, \ldots, m\}
\end{aligned}
$$

and

$$
\begin{aligned}
\mathcal{F}_{r}\left(A_{1}, \ldots, A_{m}, b\right):= & \left\{X \in \mathcal{F}\left(A_{1}, \ldots, A_{m}, b\right) \mid\right. \\
& \operatorname{rank}(X)=r\} .
\end{aligned}
$$

Proposition 2.1: Let $A_{i} \in \mathcal{S}_{+}^{d}(i=1, \ldots, m)$. Then the set $\mathcal{F}\left(A_{1}, \ldots, A_{m}, 0\right)$ has a non-zero feasible matrix with a distinct largest eigenvalue if and only if $\mathcal{F}_{1}\left(A_{1}, \ldots, A_{m}, 0\right)$ is feasible.

Proof: Let $0 \neq X \in \mathcal{F}\left(A_{1}, \ldots, A_{m}, 0\right)$. If rank $X=$ 1 , we are done. Otherwise, let $\tilde{X}:=1 / \lambda_{n}(X) X$, where $\lambda_{n}(X)$ denotes the distinct largest eigenvalue of the matrix $X$; by assumption $\lambda_{r}(X)>0$. The spectrum of $\widetilde{X}$ thus appears as

$$
\underbrace{\lambda_{1}(\tilde{X}) \leq \lambda_{2}(\tilde{X}) \leq \ldots \leq \lambda_{n-1}(\tilde{X})}_{<1}<\underbrace{\lambda_{n}(\tilde{X})}_{=1} .
$$

We note that for all $k \geq 1$, the matrices $\widetilde{X}^{k}$ belong to $\mathcal{F}\left(A_{1}, \ldots, A_{m}, 0\right)$. This follows from the observation that if $\widetilde{X} \in \mathcal{S}_{+}^{d}$ then $\widetilde{X}^{k} \in \mathcal{S}_{+}^{d}$, and automatically, $\left\langle A_{i}, \tilde{X}^{k}\right\rangle \geq 0$, given that $A_{i} \in \mathcal{S}_{+}^{d}$ for all $i$. Since $\mathcal{F}\left(A_{1}, \ldots, A_{m}, 0\right)$ is closed, it contains the limit of the sequence $\widetilde{X}^{1}, \widetilde{X}^{2}, \ldots, \widetilde{X}^{k}, \ldots$, , as $k \rightarrow \infty$; denote this limiting matrix by $X^{*}$. One has $\lambda_{1}\left(\widetilde{X}^{k}\right), \ldots, \lambda_{n-1}\left(\widetilde{X}^{k}\right) \rightarrow 0$ as $k \rightarrow \infty$, since $\lambda_{j}\left(\widetilde{X}^{k}\right)=\lambda_{j}(\widetilde{X})^{k}$ for all $j$ and $k \geq 1$. Consequently, $X^{*} \in \mathcal{F}\left(A_{1}, \ldots, A_{m}, 0\right)$ and rank $X^{*}=1$.

When the definite-ness assumption on the matrices $A_{i}$ is relaxed, an extra qualification is needed to guarantee the existence of a matrix of a given rank.

Proposition 2.2: Let $A_{i} \in \mathcal{S}^{d}(i=1, \ldots, m)$ be given such that $\mathcal{F}\left(A_{1}, \ldots, A_{m}, 0\right)$ has a non-trivial feasible point. Then for any $r \in\{1, \ldots, n\}$, the set $\mathcal{F}_{r}\left(A_{1}, \ldots, A_{m}, 0\right)$ is non-empty if $\sum_{i=1}^{m} \eta_{-}\left(A_{i}\right) \leq n-r$, where $\eta_{-}(A)$ is the number of negative eigenvalues of the matrix $A$.

Proof: Since $X, A_{i} \in \mathcal{S}^{d}(i=1, \ldots, m)$, there exist orthogonal matrices $P_{i}(i=1, \ldots, m)$ and $Q$ such that $A_{i}=P_{i} D_{i} P_{i}^{T}$ and $X=Q \Lambda Q^{T}$, where $D_{i} \quad(i=$ $1, \ldots, m)$ and $\Lambda$ are diagonal matrices whose elements are the eigenvalues of the matrices $A_{i}$ 's and $X$, respectively, ordered in the non-increasing manner. Thus

$$
D_{i}=\text { Diag }\left(d_{i 1}, \ldots, d_{i n}\right), \quad \text { and } \quad \Lambda=\operatorname{Diag}\left(\lambda_{1}, \ldots, \lambda_{n}\right),
$$

and

$$
\begin{aligned}
\left\langle A_{i}, X\right\rangle & =\left\langle P_{i} D_{i} P_{i}^{T}, Q \Lambda Q^{T}\right\rangle \\
& =\left\langle D_{i}, P_{i}^{T} Q \Lambda\left(P_{i}^{T} Q\right)^{T}\right\rangle .
\end{aligned}
$$

If $X \in \mathcal{F}_{r}\left(A_{1}, \ldots, A_{m}, 0\right)$, then one has $\Lambda=$ Diag $\left(\lambda_{1}, \ldots, \lambda_{r}, 0, \ldots, 0\right)$, with $\lambda_{i}>0$ for $i=1, \ldots, r$. Moreover, expanding (2.3) and setting the resulting expression to be nonnegative, leads to the inequality

$$
\begin{array}{r}
d_{i 1}\left\{\lambda_{1}\left(p_{i 1}^{T} q_{1}\right)^{2}+\ldots+\lambda_{r}\left(p_{i 1}^{T} q_{r}\right)^{2}\right\}+\ldots \\
+d_{i n}\left\{\lambda_{1}\left(p_{i n}^{T} q_{1}\right)^{2}+\ldots+\lambda_{r}\left(p_{i n}^{T} q_{r}\right)^{2}\right\} \geq 0,
\end{array}
$$

where $p_{i j}$ 's and $q_{j}$ are the $j$-th column of the matrices $P_{i}(i=1, \ldots, m)$ and $Q$, respectively. Now, suppose that $d_{i j}<0$; in order to satisfy the inequality (2.4) one can set $d_{i j}\left\{\lambda_{1}\left(p_{i j}^{T} q_{1}\right)^{2}+\ldots+\lambda_{r}\left(p_{i j}^{T} q_{r}\right)^{2}\right\}=0$, which is possible if $p_{i j}^{T} \perp q_{k}$ for $k=1, \ldots, r$. If there are $\kappa:=\sum_{i=1}^{m} \eta_{-}\left(A_{i}\right)$ 
such $d_{i j}{ }^{\prime} s$ for all $i, j$, then we can always find the required vectors $q_{k}$ provided that $\kappa<n$. Furthermore, since $Q$ is an orthogonal matrix, finding the required vectors $q_{k}$ $(k=1, \ldots, r)$ to construct $Q$ is feasible if $\kappa+r-1<n$; this last observation completes the proof of the proposition.

We provide two more results in this direction.

Proposition 2.3: Let $A_{i} \in \mathcal{S}^{d}$ and $b_{i} \geq 0(i=1, \ldots, m)$ be given such that the corresponding set $\mathcal{F}\left(A_{1}, \ldots, A_{m}, b\right)$ (2.1) has a non-trivial feasible point. Then for any $r \in$ $\{1, \ldots, n\}$, the set $\mathcal{F}_{r}\left(A_{1}, \ldots, A_{m}, b\right)$ is non-empty if $\sum_{i=1}^{m} \eta_{-}\left(A_{i}\right) \leq n-r$, where $\eta_{-}(A)$ is the number of negative eigenvalues of the matrix $A$. Consequently, $\max _{X \in \mathcal{F}} \operatorname{rank} X \geq n-\sum_{i=1}^{m} \eta_{-}\left(A_{i}\right)$.

Let us denote by $\eta_{+}(A)$ the number of positive eigenvalues of the matrix $A$ (as before, $\eta_{-}(A)$ will denote the number of negative eigenvalues of $A$ ).

Proposition 2.4: Let $A_{i} \in \mathcal{S}^{d}$ and $b_{i}<0(i=1, \ldots, m)$. Then there exists a nonzero matrix $X \in \mathcal{F}\left(A_{1}, \ldots, A_{m}, b\right)$ satisfying rank $X \leq r$, where

$$
r:=\min \left\{\max _{i=1, \ldots, m} \eta_{+}\left(A_{i}\right), \max _{i=1, \ldots, m} \eta_{-}\left(A_{i}\right)\right\} ;
$$

moreover, such a matrix can be obtained by solving the following SDP:

$$
\min _{X}\left\langle I_{d}, X\right\rangle
$$

subject to

$$
\begin{aligned}
\left\langle A_{i}, X\right\rangle & \geq b_{i} \quad(i=1, \ldots, m), \\
\left\langle I_{d}, X\right\rangle & >0 \\
X & \geq 0 .
\end{aligned}
$$

Proof: We provide a proof for the case where $r \leq \eta_{+}\left(A_{i}\right)$; the case of $\eta_{-}$can be proved analogously. Note that the constraint $\left\langle I_{d}, X\right\rangle>0$ ensures $X \neq 0$. Now, suppose that the rank of the optimal solution of the SDP (2.5)-(2.8) is $k>r$. Let $A_{i}=P_{i} D_{i} P_{i}^{T}$ and $X=Q \Lambda Q^{T}$, where $P_{i}(i=1, \ldots, m)$ and $Q$ are orthonormal matrices, and $D_{i}=D_{i r}^{+}+D_{i(n-r)}^{-}=\operatorname{Diag}\left(d_{i 1}, \ldots, d_{i d}\right)$, with $D_{i r}^{+}=\operatorname{Diag}\left(d_{i 1}, \ldots, d_{i r}, 0, \ldots, 0\right) \geq 0, \quad D_{i(n-r)}^{-}=$ Diag $\left(0, \ldots, 0, d_{i(r+1)}, \ldots, d_{i d}\right) \leq 0$, and $\Lambda=$ Diag $\left(\lambda_{1}, \ldots, \lambda_{k}, 0, \ldots, 0\right)$. One can assume, without loss of generality, that the matrices $A_{i}$ 's $(i=1, \ldots, m)$ admit the following decomposition

$$
A_{i}=P_{i}\left[\begin{array}{cc}
D_{i r}^{+} & 0_{r \times(d-r)} \\
0_{(d-r) \times r} & D_{i(d-r)}^{-}
\end{array}\right] P_{i}^{T} \quad \text { for all } i,
$$

where $r=\max _{i=1, \ldots, m} \eta_{+}\left(A_{i}\right)$. This assumption can be justified by the following observation: define the new matrices

$$
\widetilde{X}=\left[\begin{array}{cc}
X & X_{12} \\
X_{12}^{T} & X_{22}
\end{array}\right], \quad \widetilde{A}_{i}=\left[\begin{array}{cc}
A_{i} & 0_{d \times p} \\
0_{p \times d} & 0_{p \times p}
\end{array}\right],
$$

where $X_{12} \in \mathbf{R}^{d \times p}, X_{22} \in \mathbf{R}^{p \times p}, \tilde{X} \in \mathbf{R}^{(d+p) \times(d+p)}$, and the parameter $p$ is chosen such that

$$
\widetilde{A}_{i}=\widetilde{P}_{i}\left[\begin{array}{cc}
D_{i r}^{+} & 0_{r \times(d+p-r)} \\
0_{(d+p-r) \times r} & D_{i(d+p-r)}^{-}
\end{array}\right] \widetilde{P}_{i}^{T} \quad \text { for all } i .
$$

Note that $\widetilde{P}_{i}(i=1, \ldots, m)$ are $(d+p) \times(d+p)$ orthonormal matrices. Since

$$
\left\langle\widetilde{A}_{i}, \tilde{X}\right\rangle=\left\langle A_{i}, X\right\rangle \quad \text { and } \quad \operatorname{rank} \tilde{X} \geq \operatorname{rank} X,
$$

one can always extract a matrix $X \in \mathcal{F}\left(A_{1}, \ldots, A_{m}, b\right)$ from the matrix $\widetilde{X}$ with rank less or equal to $r$. Additionally, pertaining to the $\operatorname{SDP}(2.5)-(2.8)$, as $\widetilde{X} \in \mathcal{S}_{+}^{n+p}$, the constraint $\left\langle I_{n}, \widetilde{X}_{\alpha \alpha}\right\rangle>0$ with a suitable $\alpha \in\{1, \ldots, n\}$ can replace the original constraint (2.8). Thereby, in view of the decomposition (2.9), one has that the equivalence

$$
\left\langle A_{i}, X\right\rangle \geq b_{i} \quad \text { if and only if }\left\langle R_{i}^{T} D_{i} R_{i}, \Lambda\right\rangle \geq b_{i},
$$

where $R_{i}=P_{i}^{T} Q$. Now define $R_{i}=\left[\begin{array}{ll}R_{i 11} & R_{i 12} \\ R_{i 21} & R_{i 22}\end{array}\right]$, $D_{i}=\left[\begin{array}{cc}D_{i 1} & 0 \\ 0 & D_{i 2}\end{array}\right]$, and $\Lambda=\left[\begin{array}{cc}\Lambda_{1} & 0 \\ 0 & \Lambda_{2}\end{array}\right]$, where $R_{i 11}$, $D_{i 1}, \Lambda_{1} \in \mathbf{R}^{r \times r}, R_{i 12} \in \mathbf{R}^{r \times(d-r)}, R_{i 21} \in \mathbf{R}^{(d-r) \times r}$, and $R_{i 22}, D_{i 2}, \Lambda_{2} \in \mathbf{R}^{(d-r) \times(d-r)}$. One has $D_{i 1} \geq 0, \Lambda_{1} \geq 0$, $\Lambda_{2} \geq 0$, and $D_{i 2} \leq 0(i=1, \ldots, m)$. It can then be shown that

$$
\begin{aligned}
\left\langle A_{i}, X\right\rangle & =\left\langle R_{i}^{T} D R_{i}, \Lambda\right\rangle \\
& \geq\left\langle R_{i 21}^{T} D_{i 2} R_{i 21}, \Lambda_{1}\right\rangle+\left\langle R_{i 22}^{T} D_{i 2} R_{i 22}, \Lambda_{2}\right\rangle .
\end{aligned}
$$

Now consider the parametrized rank $r$ matrix $X_{\rho}^{*}$ defined by

$$
X_{\rho}^{*}=Q\left[\begin{array}{cc}
\Lambda_{1}^{*} & 0 \\
0 & \Lambda_{2}^{*}
\end{array}\right] Q^{T}=Q\left[\begin{array}{cc}
\rho \Lambda_{1} & 0 \\
0 & 0
\end{array}\right] Q^{T} ;
$$

we proceed to find a positive constant $\rho$ such that $X_{\rho}^{*} \in$ $\mathcal{F}\left(A_{1}, \ldots, A_{m}, b\right)$. In fact, it suffices to show that for some $\rho$,

$$
\begin{aligned}
\left\langle A_{i}, X_{\rho}^{*}\right\rangle & \geq\left\langle R_{i 21}^{T} D_{i 2} R_{i 21}, \Lambda_{1}^{*}\right\rangle+\left\langle R_{i 22}^{T} D_{i 2} R_{i 22}, \Lambda_{2}^{*}\right\rangle \\
& \geq b_{i}(i=1, \ldots, m) .
\end{aligned}
$$

Since

$$
\begin{aligned}
\left\langle R_{i 21}^{T} D_{i 2} R_{i 21}, \Lambda_{1}^{*}\right\rangle+ & \left\langle R_{i 22}^{T} D_{i 2} R_{i 22}, \Lambda_{2}^{*}\right\rangle \\
= & \left\langle R_{i 21}^{T} D_{i 2} R_{i 21}, \rho \Lambda_{1}\right\rangle \\
= & \rho\left\langle R_{i 21}^{T} D_{i 2} R_{i 21}, \Lambda_{1}\right\rangle \geq b_{i} \\
& (i=1, \ldots, m)
\end{aligned}
$$

we observe that

$$
0<\rho \leq \frac{b_{i}}{\left\langle R_{i 21}^{T} D_{i 2} R_{i 21}, \Lambda_{1}^{*}\right\rangle}=: \beta_{i} \quad(i=1, \ldots, m) .
$$

One can let $\left\langle R_{i 21}^{T} D_{i 2} R_{i 21}, \Lambda_{1}^{*}\right\rangle \neq 0$ since the inequality (2.10) holds for any $\rho$ if $\left\langle R_{i 21}^{T} D_{i 2} R_{i 21}, \Lambda_{1}^{*}\right\rangle=0$. Setting $\rho=\min _{i=1, \ldots, m}\left\{\beta_{i}, 1\right\}$, results in having $X_{\rho}^{*} \in$ $\mathcal{F}\left(A_{1}, \ldots, A_{m}, b\right)$. Finally, we note that $\left\langle I_{d}, X_{\rho}^{*}\right\rangle=$ $\rho\left\langle I_{r}, \Lambda_{1}\right\rangle<\left\langle I_{d}, X\right\rangle=\left\langle I_{r}, \Lambda_{1}\right\rangle+\left\langle I_{d-r}, \Lambda_{2}\right\rangle$, contradicting the assumption that $X$ is of minimal trace in SDP (2.5)(2.8). Hence the non-trivial optimal solution of the SDP (2.5)-(2.8) has to satisfy rank $X^{*} \leq r$; this completes the proof of the proposition. 


\section{AN ALGORITHM FOR GENERAL RMPS}

In this section we consider rank-constrained linear matrix inequalities of the form

$$
\begin{aligned}
\mathcal{P}:\left\langle A_{i}, X\right\rangle & \geq b_{i} \text { for } i=1, \ldots, m, \\
\operatorname{rank} X & =r \\
X & \in \mathcal{S}_{+}^{d},
\end{aligned}
$$

where $r \leq n$ and $A_{i} \in \mathcal{S}^{d}$ and $b_{i} \in \mathbf{R}$, for $i=1, \ldots, m$. Note note that the program $\mathcal{P}$ yields a solution to the corresponding RMP by an iteration on the parameter $r$ in (3.12). Our first result serves as a bridge between such matrix inequalities and the more familiar, yet still difficult to solve, nonconvex quadratically constrained quadratic programs.

Proposition 3.1: The problem $\mathcal{P}$ (3.11)-(3.13) is equivalent to the following nonconvex quadratically constrained quadratic program:

$$
\begin{aligned}
\mathcal{Q P}: \quad z^{T} \bar{A}_{i} z & \geq \bar{b}_{i}, \quad \text { for } i=1,2, \ldots, m, \\
z^{T} C_{j k} z & =0, \text { for } j, k=1,2, \ldots, r(j>k),
\end{aligned}
$$

where $\bar{A}_{i}=\operatorname{Diag}\left(A_{i}, A_{i}, \ldots, A_{i}\right) \in \mathbf{R}^{(r d) \times(r d)}$, and $C_{j k}$ is a block-partitioned zero matrix with $I_{d}$ in the $(j, k)$ and $(k, j)$ blocks.

Proof: When rank $X=r$, we can write $X=q_{1} q_{1}^{T}+$ $q_{2} q_{2}^{T}+\ldots+q_{r} q_{r}^{T}$, where $q_{i}^{T} q_{j}=0\left(q_{i} \in \mathbf{R}^{d}\right), i, j=$ $1, \ldots, r(i \neq j)$. Then $\left\langle A_{i}, X\right\rangle=q_{1}^{T} A_{i} q_{1}+q_{2}^{T} A_{i} q_{2}+\ldots+$ $q_{r}^{T} A_{i} q_{r}$; now let $z=\left[q_{1}^{T}, q_{2}^{T}, \ldots, q_{r}^{T}\right]^{T}$ and observe the stated equivalence between $\mathcal{P}$ and $\mathcal{Q P}$.

\section{A. The algorithm}

We will now propose an algorithm for finding a feasible matrix for problem $\mathcal{P}$ (3.11)-(3.13), or in view of Proposition 3.1, a feasible vector for problem $\mathcal{Q P}$ (3.14)(3.14). In this venue we will first identify the norm of a candidate solution satisfying the constraints in $\mathcal{Q P}$. Then all the nonconvex constraints in $\mathcal{Q P}$ are re-parametrized in terms of convex constraints augmented with a norm constraint. The proposed procedure then essentially generate a sequence of vectors satisfying the convex constraints while asymptotically assuming the prescribed norm.

Let us start by observing that each nonconvex quadratic constraints in $\mathcal{Q P}$ (3.14)-(3.14) of the form $z^{T} \hat{A}_{i} z \geq \hat{b}_{i}$, or equivalently, $\tilde{z}^{T}\left[\begin{array}{cc}\hat{A}_{i} & 0 \\ 0 & -\hat{b}_{i}\end{array}\right] \tilde{z} \geq 0$, where $\tilde{z}=\left[\begin{array}{ll}z & 1\end{array}\right]^{T}$, can be written as,

$$
\tilde{z}^{T} \tilde{A}_{i} \tilde{z} \leq \eta
$$

where $\omega_{i}>0$ is chosen such that,

$\tilde{A}_{i}=I_{n+1}-\frac{1}{\omega_{i}} \operatorname{Diag}\left(\hat{A}_{i},-\hat{b}_{i}\right)>0 \quad$ and $\quad \eta=\tilde{z}^{T} \tilde{z}$.

As in our subsequent discussions we will only work with inequalities of the form (3.14), we write $z$ instead of $\tilde{z}$ in (3.14)-type inequalities for notational convenience. Note

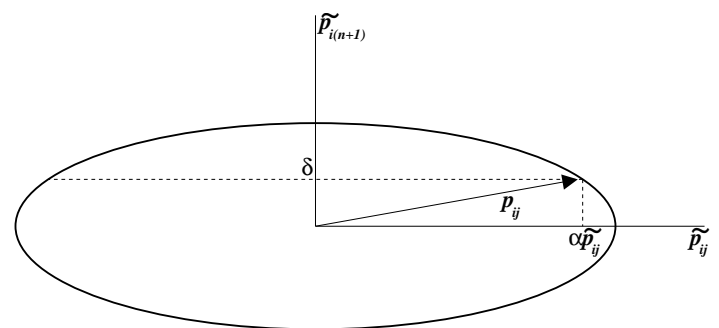

Fig. 1. The construction of the principal vector $p_{i j}$ with $\alpha>0$.

that since $\tilde{A}_{i}>0$, the inequality (3.14) defines a nondegenerate ellipsoid. We define the principal vector $p_{i j}(j=$ $1,2, \ldots, n+1)$ of the ellipsoid (3.14) with $\eta=1$, as ${ }^{1}$

$$
p_{i j}:=\left[\alpha_{i} \tilde{p}_{i j}, \delta\right]^{T},
$$

where $\alpha_{i}= \pm \sqrt{\left(1-\tilde{\lambda}_{i(n+1)} \delta^{2}\right) / \tilde{\lambda}_{i j}}, \tilde{p}_{i j}$ (respectively, $\tilde{\lambda}_{i j}$ ) is the $j$-th unit eigenvector (respectively, eigenvalue) of $\tilde{A}_{i}$, and $\delta$ is a sufficiently small constant. Figure 1 illustrates how the principal vector $p_{i j}$ is constructed. Assuming that the set defined by $\mathcal{Q P}$ (3.14)-(3.14) is nonempty, our proposed algorithm proceeds as follows:

1) Let $k=0, n=0$, and $\Omega^{(0)}=\left\{z \mid z^{T} \tilde{A}_{i} z \leq\right.$ $\left.1(i=1, \ldots, m+r(r-1)), z^{T} z \leq 1\right\}$. Moreover, find an initial vector $z_{o} \in \mathbf{R}^{r d+1} \cap \Omega^{(0)}$ where the last element of $z_{o}$ is strictly positive.

2) Solve the following convex program

$$
\mathcal{C P}: \max _{z_{n+1}} z_{n}^{T} z_{n+1}
$$

subject to

$$
\begin{aligned}
z_{n+1} & \in \Omega^{(k)}, \\
c^{T} z_{n+1} & \geq \delta,
\end{aligned}
$$

where $c=[0,0, \ldots, 0,1]^{T}$ and $\delta$ is a sufficiently small constant. If $\left\|z_{n+1}-z_{n}\right\|<\epsilon$, let $n=n+1$ and proceed with Step 3; otherwise, let $n=n+1$ and repeat solving $\mathcal{C P}$ (3.16)-(3.18).

3) If for sufficiently small $\epsilon>0$,

$$
\left\|z_{n}^{T} z_{n}-1\right\|<\epsilon
$$

terminate the algorithm.

4) Find a feasible principal vector $p$ (3.15) with $\|p\|>$ $\left\|z_{n}\right\|$ and $p^{T} z_{n} \geq 0$ such that $\bar{z}_{n} \in \Omega^{(k)}$, where

$$
\bar{z}_{n}=(1+\epsilon) \frac{(1-t) z_{n}+t p}{\left\|(1-t) z_{n}+t p\right\|} \text { for small } \epsilon, t>0 ;
$$

if such a vector $p$ exists, let $z_{n}=\bar{z}_{n}$, and proceed with Step 2. ${ }^{2}$

5) Construct a new region $\Omega^{(k+1)}$ by partitioning the current feasible region $\Omega^{(k)}$ via a hyperplane. In this venue, first identify all the feasible principal vectors

\footnotetext{
${ }^{1}$ The subscript $j$ runs up to $2(n+1)$ as each $\tilde{p}_{i j}$ essentially corresponds to two $p_{i j}$ 's.

${ }^{2}$ The vector $\bar{z}_{n}$ is feasible as the set $\Omega^{(k)}$ is convex.
} 
$p_{i}$ 's $(i=1,2, \ldots)$ with $\left\|p_{i}\right\| \leq\left\|z_{n}\right\|$ and $p_{i}^{T} z_{n} \geq 0$ such that $\bar{z}_{n} \in \Omega^{(k)}$, where

$$
\bar{z}_{n}=(1-\epsilon) \frac{(1-t) z_{n}+t p_{i}}{\left\|(1-t) z_{n}+t p_{i}\right\|} \text { for small } \epsilon, t>0 .
$$

If $c^{T} z_{n}=\delta$, where $c=[0,0, \ldots, 0,1]^{T}$, include the vector $[0,0, \ldots, 0, \delta]^{T}$ in the set of feasible principal vectors $p_{i}$ 's. If the number of linearly independent such $p_{i}$ 's is less than $r d+1$, terminate the algorithm. Construct the hyperplane $\mathcal{H}=\left\{x \mid h^{T} x=\gamma\right\}$ such that: $h^{T} z_{n}>\gamma, h^{T} p_{i}=\gamma$ for $1 \leq i \leq r d+1$, and $h^{T} p_{i} \leq \gamma$ for $i \geq r d+2$. Define

$$
\Omega^{(k+1)}:=\Omega^{(k)} \cap\left\{x \mid h^{T} x \leq \gamma\right\} ;
$$

let $k=k+1, n=n+1, z_{n}=\bar{z}_{n}$, and repeat Step 2.

Let us provide the justifications for the proposed algorithm. The first result clarifies the role of parameter $\eta$ in Step 1 of the algorithm.

Proposition 3.2: If the program $\mathcal{Q P}$ (3.14)-(3.14) is feasible, then there exists a unit magnitude feasible vector $\hat{z} \in \mathbf{R}^{r d+1}$ with the last entry $z_{d+1} \neq 0$, such that

$$
\hat{z}^{T}\left[\begin{array}{cc}
\hat{A}_{i} & 0 \\
0 & -\hat{b}_{i}
\end{array}\right] \hat{z} \geq 0, \quad i=1,2, \ldots, m .
$$

Proof: If the program $\mathcal{Q P}$ (3.14)-(3.14) is feasible, then there exists a feasible solution $z$ satisfying (3.20) and $z_{d+1} \neq 0$. As the right-hand side of (3.20) is zero, the corresponding normalized vector $\hat{z},\|\hat{z}\|=1$, is also feasible.

The next proposition clarifies the inclusion of the (3.18) in the program $\mathcal{C P}$ (3.16)-(3.18).

Proposition 3.3: If $\mathcal{Q P}$ is feasible then there exists a feasible vector $z \in \mathbf{R}^{r d+1}$ such that

$$
z \in \Omega^{(0)} \quad \text { and } \quad c^{T} z \geq \delta
$$

where $c=[0,0, \ldots, 0,1]^{T}$ and $\delta>0$ is sufficiently small.

Proof: Suppose that $z$ is a feasible for $\mathcal{Q P}$. Proposition 3.2 implies that, without loss of generality, we can assume $z \in \Omega^{(0)}$ with $\eta=1$ and $z_{d+1} \neq 0$, i.e., $\left|c^{T} z\right| \geq \delta$ for sufficiently small $\delta>0$. Since the vector $-z$ is also a feasible for $\mathcal{Q P}$, the statement of the proposition now follows.

Proposition 3.4: The sequence generated by repeatedly solving the convex program $\mathcal{C P}$ (3.16)-(3.18) converges.

Let us call the limit of the sequence generated by repeatedly solving $\mathcal{C P}$ (3.16)-(3.18) with no norm-increasing feasible directions available to it, an apex point. When the algorithm reaches such an apex point, the algorithm determines a cut in Step 5, i.e., a half space, that would exclude that apex point in the subsequent iteration of the algorithm.

Proposition 3.5: If $\mathcal{Q P}$ (3.14)-(3.14) is feasible, so is $\mathcal{C P}$ (3.16)-(3.18). Moreover, for all $k \geq 0$, one has $\operatorname{dim}\left(\Omega^{(k)}\right)=$ $r d+1$.

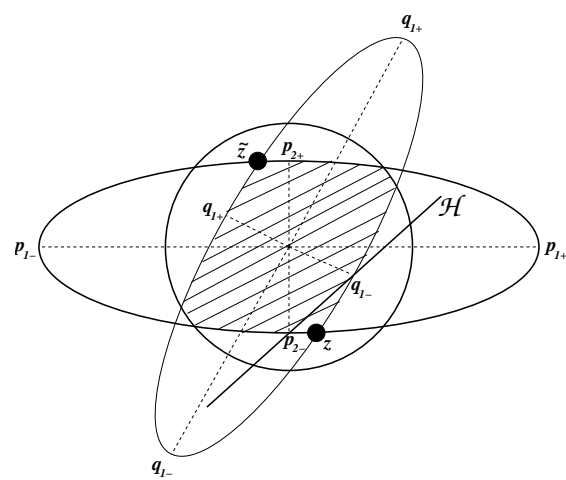

Fig. 2. Apex points $(z, \tilde{z})$, principal vectors $(p, q)$, the separating hyperplane $(\mathcal{H})$ in Step 5, and the feasible set $\Omega^{(k)}$ for some $k \geq 0$ (dashed area); $p_{2-}, q_{1-}$ are the feasible principal vectors at $z$.

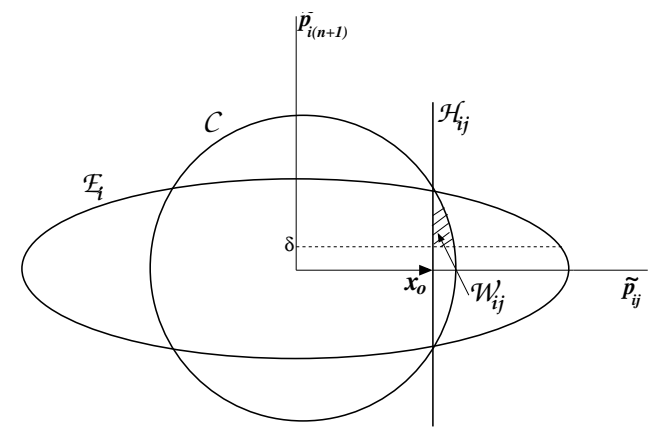

Fig. 3. The construction of the set $\mathcal{W}_{i j}$ (dashed area)

Proposition 3.6: Let $\left\{z_{n}\right\}_{n \geq 0}$ denote the sequence generated by the proposed algorithm. If $\mathcal{Q P}$ (3.14)-(3.14) is feasible then

$$
\left\|z_{n}^{T} z_{n}\right\| \rightarrow 1 \quad \text { as } \quad n \rightarrow \infty .
$$

The running time of the algorithm is proportional to the number of apex points in the feasible set of $\mathcal{C P}$ (3.16)(3.18) with $k=0$. We note that the number of apex points however can be exponential in the dimension of $\mathcal{Q P}$. Thus the proposed algorithm is not guaranteed to run in polynomial-time; after all, RMPs are NP-hard! However, as the example in $\S \mathrm{IV}$ demonstrates, the number of visited apex points is generally small as compared with the total number of principal vectors $p$ 's with $\|p\|<1$, implying that the proposed algorithm is potentially efficient in practice. We close this section with a procedure that reduces the number of apex points in the feasible set of $\mathcal{C P}$ when $k=0$. In this direction, first define the following sets $\mathcal{C}=\left\{z^{T} z \leq 1\right\} \quad(\eta>0), \mathcal{E}_{i}=\left\{z^{T} \tilde{A}_{i} z \leq 1\right\}\left(\tilde{A}_{i} \in\right.$ $\left.\mathcal{S}_{+}^{d}, i=1,2, \ldots, m\right), \mathcal{H}_{i j}=\left\{x \in \mathbf{R}^{r d+1}, \tilde{p}_{i j}^{T}\left(x-x_{o}\right) \geq\right.$ $0\}, \mathcal{W}_{i j}=\mathcal{C} \cap \mathcal{E}_{i} \cap \mathcal{H}_{i j} \cap\left\{z \mid c^{T} z \geq \delta\right\} \quad\left(j=1, \ldots, \beta_{i}\right)$, where $\tilde{p}_{i j}$ is an eigenvector vector- which corresponds to an eigenvalue greater than or equal to 1 - of the matrix $\tilde{A}_{i}$, $\beta_{i}$ is the number of such $\tilde{p}_{i j}$ 's for each $i, x_{o}$ is chosen such that $\mathcal{H}_{i j}$ contains $r d+1$ many intersection points of $\mathcal{C}, \mathcal{E}_{i}$, and $\mathcal{H}_{i j}$ (see Figure 3), and $c=[0,0, \ldots, 1]^{T}$.

Proposition 3.7: If there exists a feasible solution $z$ of 


\begin{tabular}{|c|c|c|c|c|c|}
\hline \multirow[b]{2}{*}{$d$} & \multirow[b]{2}{*}{$r$} & \multirow[b]{2}{*}{ \# of constraints } & \multicolumn{2}{|c|}{$\#$ of principal vectors $p$} & \multirow[b]{2}{*}{ \# of cuts } \\
\hline & & & $\|p\| \geq 1$ & $\|p\|<1$ & \\
\hline 3 & 2 & 8 & 82 & 30 & 1 \\
\hline 4 & 3 & 18 & 372 & 96 & 1 \\
\hline 5 & 2 & 22 & 396 & 88 & 1 \\
\hline 5 & 4 & 32 & 1124 & 220 & 2 \\
\hline 6 & 5 & 50 & 2680 & 420 & 1 \\
\hline 10 & 2 & 92 & 3540 & 324 & 2 \\
\hline
\end{tabular}

TABLE I

THE TOTAL NUMBER OF CONSTRAINTS, PRINCIPAL VECTORS, AND CUTS (APEX POINTS VISITED) FOR VARIOUS VALUES OF $d$ AND $r$

$\mathcal{Q P}$ (3.14)-(3.14) then for any $i$, there exists an index $j$ such that $z \in \mathcal{W}_{i j}$.

Proposition 3.7 states that one can find a feasible solution of $\mathcal{Q P}$ by starting the algorithm from the region $\mathcal{W}_{i j}$, for fixed $i$ and $j$, instead of $\Omega^{(0)}$ - and then repeating the algorithm with $\mathcal{W}_{i j}$ for different index $j$ at most $\beta_{i}$ times. This parsing technique on the other hand can significantly reduce the number of apex points visited by the procedure.

\section{AN EXAMPLE}

We now provide an numerical example for the graph realization problem as formulated in terms of rank-constrained matrix inequalities. The graph realization problem can be stated as follows [1], [2]: suppose that we are given a weighted graph $G=(V, E ; \rho)$, where $V=\left\{v_{1}, v_{2}, \ldots, v_{d}\right\}$ is the vertex set, $E$ is the edge set, and $\rho: E \rightarrow \mathbf{R}_{+}$ assigns to every edge $(i, j) \in E$ a non-negative number $\rho_{i j}=\left\|v_{i}-v_{j}\right\|$. We wish to verify whether $v_{1}, v_{2}, \ldots, v_{d}$ can be configured in $\mathbf{R}^{r}$, i.e., examining if it is $r$-realizable. The graph realization problems can be formulated in terms of finding an $n \times n$ matrix $X=\left[x_{i j}\right]$ such that $x_{i j}-$ $2 x_{i j}+x_{j j}=\rho_{i j}^{2}$, for all $(i, j) \in E$, while $X \in \mathcal{S}_{+}^{d}$ and rank $X=r$. For the purpose of measuring the numerical performance of the proposed algorithm, we considered a worst case $r$-realizable graph and compared the numbers of apex points visited during the execution of the proposed algorithm for various values of $d, r$. Table I indicates how the number of principal vectors employed in the graph realization problem grows as the parameters $d$ or $r$ are increased. However, even in the presence of many principal vectors with magnitude less than 1 , the number of apex points visited in our numerical examples was at most 2 .

\section{REFERENCES}

[1] A. Barvinok. A remark on the rank of positive semidefi nite matrices subject to affi ne constraints, Discrete and Computational Geometry, 25:23-31, 2001.

[2] A. Barvinok. A Course in Convexity. American Mathematical Society, Providence, RI, 2002.

[3] E. Beran. Methods for Optimization-based Fixed-Order Control Design, Ph. D. Dissertation, Technical University of Denmark, 1997.

[4] S. P. Boyd, L. El Ghaoui, E. Feron, and V. Balakrishnan. Linear Matrix Inequalities in System and Control Theory, SIAM, Philadelphia, 1994.

[5] J. F. Buss, G. S. Frandsen, and J. O. Shallit. The computational complexity of some problems of linear algebra, BRICS Report Series, 1996.
[6] J. David. Algorithms for Analysis and Design of Robust Controllers, $\mathrm{Ph}$. D. Dissertation, Katholieke Universiteit Leuven, 1994.

[7] A. d'Aspremont. A semidefi nite representation for some minimum cardinality problems, Technical Report, Stanford University, 2003.

[8] M. Deza and M. Laurent. Geometry of Cuts and Metrics, SpringerVerlag, 1997.

[9] L. El Ghaoui and P. Gahinet. Rank minimization under LMI constraints: a framework for output feedback problems, European Control Conference, July 1993.

[10] M. Fazel, H. Hindi, and S. Boyd. A rank minimization heuristic with application to minimum order system approximation, American Control Conference, June 2001.

[11] M. Fazel. Matrix Rank Minimization with Applications, Ph. D. Dissertation, Stanford University, California, 2002.

[12] M. Fu and Z. Q. Luo. Computational complexity of a problem arising in fi xed order output feedback design, Systems and Control Letters, 30:209-215, 1997.

[13] J. F. Geelen. Maximum rank matrix completion, Linear Algebra and its Applications, 288:211-217, 1999.

[14] L. El Ghaoui, F. Oustry, and M. AitRami. A cone complementarity linearization algorithm for static output-feedback and related problems. IEEE International Symposium on Computer-Aided Control System Design, Michigan, 1996.

[15] G. Golub and C. van Loan. Matrix Computations. Johns Hopkins University Press, 1996.

[16] K. Grigoriadis and R. Skelton. Fixed-order design for LMI control problems using alternating projection methods, IEEE Conference on Decision and Control, December 1994.

[17] L. Gurvits and M. Mesbahi. A polynomial-time algorithm for certain classes of rank minimization problem. American Control Conference, June 1998.

[18] A. Hassibi, J. P. How, and S. P. Boyd. A path-following method for solving BMI problems in control. American Control Conference, June 1999.

[19] D. Henrion, S. Tarbouriech, and M. Sebek, Rank-one LMI approach to simultaneous stabilization of linear systems, Systems and Control Letters, 38:79-89, 1999.

[20] D. Henrion. Low-order robust controller synthesis for interval plants, International Journal of Control, 74:1-9, 2001.

[21] S. Ibaraki. Rank minimization approach for solving BMI problems with random search, American Control Conference, June 2001.

[22] T. Iwasaki. The dual iteration for fi xed-order control, IEEE Transactions on Automatic Control, 44 (4):783-788, 1999.

[23] C. R. Johnson. Matrix completion problems: a survey. Proceedings of Symposia in Applied Mathematics, 40:171-198, 1990.

[24] Y. Kim and M. Mesbahi. Quadratically constrained spacecraft attitude control via semidefi nite programming, IEEE Conference on Decision and Control, December 2003.

[25] W. Ledermann. On a problem concerning matrices with variable diagonal elements, Proceedings of the Royal Society of Edinburgh, Vol. LX, 1939-1940.

[26] M. Mesbahi and G. P. Papavassilopoulos. On the rank minimization problem over a positive semi-defi nite linear matrix inequality, IEEE Transactions on Automatic Control, 42 (2):239-243, 1997.

[27] M. Mesbahi. On the rank minimization problem and its control applications, Systems and Control Letters, 33:31-36, 1998.

[28] Y. Nesterov and A. Nemirovskii. Interior-Point Polynomial Algorithms in Convex Programming, SIAM, Philadelphia, 1994.

[29] J. B. Lasserre. An explicit equivalent positive semidefi nite program for nonlinear 0-1 programs, SIAM Journal on Optimization, 11:756$769,2002$.

[30] P. M. Nylen. Minimum-rank matrices with prescribed graph, Linear Algebra and its Applications, 248:303-316, 1996.

[31] P. A. Parrilo and S. Khatri. On cone-invariant linear matrix inequalities, IEEE Transactions on Automatic Control, (45) 8: 1558-1563, 2000.

[32] M. Ramana. An Algorithmic Analysis of Multiquadratic and Semidefinite Programming Problem, Ph. D. Dissertation, Johns Hopkins University, Maryland, 1993.

[33] R. E. Skelton, T. Iwasaki, and K. Grigoriadis, A Unified Algebraic Approach to Linear Control Design, Taylor and Francis, 1998.

[34] V. Syrmos, C. Abdallah, P. Dorato, and K. Grigoriadis. Static output feedback, Automatica, 33(2):125-137, 1997. 